\title{
Une "femme machine" au travail : la "demoiselle du téléphone"
}

Virginie Julliard

\section{Citer ce document / Cite this document :}

Julliard Virginie. Une "femme machine" au travail : la "demoiselle du téléphone". In: Quaderni, n56, Hiver $2004 / 2005$. Agriculture et technologies. pp. 23-32.

doi : 10.3406/quad.2004.1645

http://www.persee.fr/doc/quad_0987-1381_2004_num_56_1_1645

Document généré le 17/10/2015 


\section{Communication}

\section{une "femme} machine"

la "demoiselle du téléphone"

\section{Virginie Julliard}

Doctorante en

Information Communication à l'Institut Français de Presse Vacataire à l'Université de Compiègne
$\mathrm{Au} \mathrm{XIX}^{\mathrm{c}}$ siècle, l'être humain ne considère plus la machine avec la même confiance qu'au siècle précédent. Certes, les nouveaux objets techniques mus par l'électricité suscitent l'émerveillement du grand public qui se presse aux expositions universelles et dans les salons, mais l'introduction massive des machines dans le processus de production génère des tensions dans les rangs des ouvriers. Au milieu du siècle, le marxisme dénonce les effets néfastes des machines sur la valeur du travail, la déqualification des métiers, la pression sur les salaires. Cet affrontement de l'homme et de la machine trouve de nombreuses expressions littéraires et iconographiques. Dans $L$ 'Ève future, Villiers de L'Isle-Adam exprime la fascination et la crainte que lui inspirent les avancées de la science. Zola développe dans son œuvre l'idée "que le fer se nourrit de la chair", de la sueur et du sang des hommes. Selon Jacques Noiray, "cette violence maléfique de la machine revêt, suivant le sexe sur lequel elle s'exerce, des caractères différents"'. Pour l'homme, cette violence se traduit par l'aliénation du travail. Les rapports que la machine entretient avec la femme sont marqués par la violence du détournement de la femme de sa finalité reproductive. On admet d'une manière générale que femme et machine s'opposent selon la dichotomie nature/artifice. Aussi le travail féminin sur machine, à l'extérieur de la sphère domestique, équivaut à la négation par l'ouvrière de sa destination naturelle. Ce refus, causé par la nécessité, porte atteinte à la moralité de l'individu féminin.

Marqué par une conversation téléphonique qu'il eut avec sa mère en 1896, Marcel Proust évoque à plusieurs reprises le lien désincarné que tisse le téléphone entre les interlocuteurs, et entre les 
interlocuteurs et les employées. Il contribue en cela à la construction d'un imaginaire fécond autour du téléphone et de ses demoiselles, témoignant de l'instrumentalisation des téléphonistes et de l'érotisation de l'objet de communication. Sa description de l'enchevêtrement des représentations de la femme et du téléphone esquisse une image féminine, anarchique, du réseau de communication.

\section{Aliénation de l'employée du téléphone}

Le récit autobiographique de Madeleine Campana, jeune demoiselle du téléphone au début du $\mathrm{XX}^{\mathrm{c}}$ siècle, nous livre un capital de savoir sur la symbolique sociale et technique du téléphone.

"Je deviens un robot, un appareil ménager, précisément, placé devant un autre robot "2. Dans La Demoiselle du téléphone, Madeleine Campana retrace sa carrière au sein de l'administration des PTT où elle est affectée au service du téléphone entre 1921 à 1934. Elle y évoque les gestes quotidiens de la demoiselle au temps de la commutation manuelle. Responsable de cent abonnés représentés sur son "multiple" par de petites lampes, la téléphoniste est chargée d'établir les communications qu'ils reçoivent ou souhaitent passer. "Nous répondons à toute allure aux injonctions lumineuses du tableau, nous passons le bras l'une par dessus la tête de l'autre, nous nous levons, nous nous rasseyons,

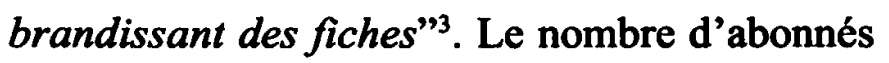
et la fréquence des appels sont en constante augmentation. En 1903, les dames des postes adressent au Parlement un mémoire dans lequel elles indiquent qu'en moyenne, chaque employée traite 6 communications par minute. Elles témoignent également de l'aspect déshumanisant de leur emploi dans les 24 commandements de la téléphoniste, contestant l'obéissance irraisonnée aux ordres et évoquant le surmenage d'un tel service 4 . Les corps des demoiselles, répondant aux injonctions de la machine, pliés à des cadences épuisantes, sont également contraints par des "harnachements". Elles portent des casques lourds et parlent dans des microphones placés devant leur bouche, prothèses de leur ouïe et de leur voix. "Mes gestes mal assurés de débutante sont gênés par le harnachement dont je n'ai pas l'habitude [...] Le casque, la prise qui me relie au standard par une mâchoire, l'espèce d'instrument que j'ai devant la bouche pour lancer mes " $J$ 'écoute": il tient à la fois de l'entonnoir, de l'embouchoir pour instruments à vent et du cornet acoustique. [...] Cet attirail est pesant : dans mon dos, un contre-poids maintenu par des sangles $m$ 'aide à remonter et redescendre le cornet suivant mes manceuvres"s. Pour être sédentaire, cet emploi n'en exige pas moins une dépense considérable et une subordination de l'employée à la machine qu'elle actionne. L'expression même de "demoiselle du téléphone" suggère presque sa dépossession au profit de l'être d'acier auquel elle confère tous ses soins.

L'administration ajoute aux contraintes de productivité, une contrainte morale. Pour répondre aux attaques des opposants au travail féminin, qui estiment que la machine pervertit les organes reproductifs de la femme, les industricls et l'administration la présentent comme un outil de domestication des corps. Pour convaincre de l'utilité des travailleuses, ils appuient leurs discours sur les qualités naturelles de la femme 
qui trouvent un emploi tout particulier dans les tâches d'exécution. L'étude du discours de l'administration, et des représentations des demoiselles révèle "l'idéologie domestique" qui les structure. Le travail féminin fait une place particulière au corps de la femme. Sa présentation sobre (les employées des PTT sont vêtues de noir) doit traduire docilité, décence et retenue. "Les gestes du travail féminin mêlent exigences techniques et code de discipline". Marcel Proust, cité avec ferveur par Madeleine Camapana qui estime qu'il est le seul à dessiner un imaginaire positif de sa profession, développe à plusieurs reprises le thème de la confidence téléphonique. Dans la scène du "téléphonage" à Doncières, qui s'inscrit au cour du premier tome du Côté de Guermantes publié en $1920^{8}$, l'écrivain fait implicitement référence à la symbolique des moires, des fileuses. Le tissage et la couture sont envisagés comme des vecteurs de la domestication du corps féminin. Symboles de la féminité, ils font partie intégrante de l'éducation des jeunes filles, et sont considérés comme un "temps de l'auto-formation affective et sexuelle". La machine à coudre, proclamée "machine de la mère"10, est placée aussi bien à la maison qu'à l'usine. Femme et machine ne sont pas incompatibles dans certains secteurs traditionnellement reconnus comme féminins. Le service du téléphone, travaillé chez Proust par la métaphore du tissage, semble faire partie de ces métiers féminins qui initient la femme à sa destinée. Le célibat et la preuve d'une existence honorable, à apporter lors de l'inscription au concours d'entrée, d'une part, la stricte réglementation du mariage et le jeune âge des employées, d'autre part, renforcent cette idée de formation féminine. Dans les journaux de l'administration, peu d'employés contestent le service "féminin" du téléphone. Considéré comme un emploi statique, il semble mieux convenir aux femmes et libère les hommes pour des emplois plus adaptés à leurs capacités : postes à responsabilité, emplois indépendants et nécessitant une dépense physique. En réalité, la division sexuelle du travail fait partie des mesures de rationalisation du travail, et doit donc se comprendre comme le déni d'une qualification du travail féminin et une stratégie de réduction du coût de la main d'œuvre.

\section{Érotisation de la machine}

Selon Jacques Noiray, la machine apparaît fréquemment comme un objet érotique dans la littérature du $\mathrm{XIX}^{\mathrm{e}}$ siècle. D'une part, les romanciers l'humanisent, lui prêtent un corps, des attributs physiques, et d'autre part, elle se caractérise par des mouvements de va et vient. "Erotisé, l'objet technique sera le plus souvent sexualisé, c'est-à-dire, dans l'imagination de l'auteur et de ses personnages mâles, féminisé". Certaines machines sont clairement sexuées, c'est le cas du train "équivalent symbolique du sexe masculin" ", d'autres se "sexualisent" en fonction des usages qu'on leur attribue.

Objet de transmission de la parole, le téléphone ne "possède" pas de corps, mais il en hypertrophie la voix et l'ouïe. Il ne se caractérise pas par un mouvement productif de va et vient, mais permet la circulation des communications sur le réseau téléphonique. Dans ses textes, Proust humanise le téléphone, comparant le combiné à une marionnette qui prend la voix de celle qui l'actionne, et jacasse comme le font les femmes 
oisives. Métaphores de la communication qui $s$ 'instaure grâce à elles, les demoiselles demeurent invisibles aux interlocuteurs, ce sont de pures voix désincarnées qui prêtent leurs corps à la technique, comme les oracles incarnent la parole divine, pour créer le lien.

Madeleine Campana cite, dans son autobiographie, un article de $L$ 'Intransigeant paru en 1929 à l'occasion du Salon de l'automobile. Le journaliste y compare les demoiselles, embauchées en extra à cette occasion, à des abeilles. "En face du meuble, elles semblent, menues ouvrières de la ruche, composer un gâteau de miel. Chaque alvéole reçoit leurs soins empressés. Abeilles? Elles en ont la taille"12. Le service du téléphone est un emploi féminin qui arraisonne le corps à la machine dans la fixité et la répétition intuitive, gestes utiles du travail féminin qui érotisent la travailleuse. Ici, le reporter donne pour équivalents une technique et un processus vital et naturel. Femme et machine se ressemblent dans leur exécution répétitive des choses, loin de toute transcendance.

Les représentations qui sont faites du téléphone au début du $\mathrm{XX}^{\mathrm{e}}$ siècle donnent pour équivalentes déficience technique et frivolité féminine. Si les demoiselles du téléphone échouent à transmettre la communication, c'est qu'elles y mettent de la mauvaise volonté. "Les capricieuses Gardiennes n'avaient pas voulu ouvrir les portes merveilleuses ou sans doute elles ne le purent pas..."'3.

Contrairement au télégraphe, le téléphone nationalisé en 1889 ne bénéficie pas d'un financement permettant son développement. $\mathrm{Ce}$ sont les communes qui doivent supporter le coût de la construction et du rachat des réseaux. Dans un premier temps, elles privilégient la création de centraux communaux et délaissent les liaisons interurbaines. En l'absence de décision centralisée, le réseau téléphonique du début du siècle est "anarchique". Le nombre des communications va croissant sans qu'il y ait d'investissement proportionnel. La création de l'Association des abonnés au téléphone pour l'amélioration du service téléphonique, en 1905, illustre le mécontentement des usagers. Elle publie en 1909 une brochure intitulée "Une honte nationale, l'anarchie téléphonique"14, qui dénonce le "matériel insuffisant et démodé", les "erreurs" et le "recrutement des téléphonistes". Les "ne coupez pas, mademoiselle!"'s traduisent l'assimilation, dans l'esprit des abonnés, de la téléphoniste et de la technique. Ils occultent les dysfonctionnements du réseau qu'ils attribuent au manque de sérieux des jeunes filles. Pour beaucoup, la curiosité féminine va à l'encontre du respect du secret des communications. En principe, celui-ci est absolu et respecté. Si la téléphoniste écoutait une conversation, elle serait immédiatement sanctionnée. Les demoiselles du téléphone sont également tenues au secret du fonctionnement de leur service. Elles ne peuvent révéler aux abonnés impatientés les raisons techniques qui leur ont interdit de répondre au standard. "Le mot d'ordre était [...] de cacher la véritable situation en laissant de ce fait retomber toute la responsabilité du mauvais fonctionnement sur les opératrices"16. À plusieurs reprises, Madeleine Campana évoque le devoir de discrétion inhérent à son travail, et les reproches des abonnés auprès de qui elle ne peut se justifier. Lorsqu'une dératisation, 
nécessitant l'évacuation des employées, paralyse le central, il lui est "impossible [...] de dire à [ses] chers abonnés qu'en plein $X X^{e}$ siècle, le temple du progrès a subi une moyenâgeuse invasion de rongeurs"17. Une des revendications des demoiselles du téléphone fut, très tôt, d'avoir le droit d'expliquer aux abonnés les raisons des lenteurs qui les exaspèrent.

"La société française des années 1880 assigne [au téléphone] deux fonctions traditionnelles : porter des ordres d'hommes et des bavardages de femmes"18. La lenteur de la transmission, due au mauvais entretien des lignes et à leur encombrement limite les usages professionnels. Un imaginaire fécond, focalisé sur les usages récréatifs, se construit autour du téléphone. Véhicule des conversations privées, cet instrument de confidence et de secret inspire la littérature populaire, la publicité, le cinéma.

Depuis la Contre Réforme, l'église catholique encourage la confession des péchés liés à la chair et enjoint ses fidèles à traquer le mal en eux ${ }^{19}$. Au tournant des $\mathrm{XIX}^{\mathrm{c}}$ et $\mathrm{XX}^{\mathrm{e}}$ siècles, l'examen de conscience est valorisé, tandis que l'individualisme se développe. Le téléphone est le prolongement du confessionnal et des causeuses. Il favorise la confidence, le dialogue. Il constitue également pour les femmes confinées dans leur intérieur, un moyen d'évasion, la possibilité d'avoir accès au monde extérieur. Il favorise enfin le développement du capital social de la famille qui passe par l'entretien des liens familiaux et amicaux.

Le téléphone est aussi et surtout, pour le public, un instrument de séduction, les gravures libertines qu'il inspire en témoignent et renforcent cette perception ${ }^{20}$. Dans Le côté de Guermantes, Proust identifie l'interlocuteur privilégié à la "fiancée", ou encore à "l'être cher", aux "les personnes aimées" 21 . Le téléphone est l'instrument des amoureux, parfois des femmes adultères. Ici, ce n'est plus la bouche qui livre des confidences à l'oreille attentive, mais les lèvres qui se pressent sur le combiné comme pour envoyer un baiser à l'être aimé. Tout un champ lexical de la proximité amoureuse se déploie dans le texte de Proust : "L'amie à qui nous avions le désir de parler [...] se trouve [...] contre notre oreille". C'est le "rapprochement le plus doux" que de pouvoir causer avec elle, et l'auteur souhaiterait "embrasser" les "paroles" murmurées par des "lèvres à jamais en poussière"22.

Une illustration du chapitre "communication et littérature" dans le Dictionnaire critique de la communication représente une jeune femme en vêtement d'intérieur, étendue endormie sur un lit et tenant dans sa main le combiné d'un téléphone ${ }^{23}$. La légende de l'illustration, "le cher objet", présente le téléphone comme un fétiche. Doté du don d'ubiquité, l'amant peut s'introduire dans l'intimité du foyer. Invalidée par la maladie ou la morale, la riche bourgeoise ou l'aristocrate n'est plus limitée par son enveloppe charnelle confinée dans le domaine privé. Grâce à sa "prothèse", elle reçoit virtuellement. Le fétiche, symbole d'une énergie divine captée et utilisable, condense "l'objet du désir inavouable", le rend "maniable"24. Le téléphone favorise, voire encourage, dans l'imaginaire populaire, l'infidélité. Enfin, l'invisibilité de l'interlocuteur, la voix sans le regard, laisse libre cours à l'imagination, 
véritable ressort de l'érotisme.

L'enchevêtrement des imaginaires de la femme et du téléphone, dont Proust se fait le témoin, caractérise les débuts du téléphone. L'auteur semble faire de l'origine du téléphone, un temps féminin primitif.

\section{Une image féminine du réseau de communication?}

Les réseaux, tels qu'ils sont présentés par les disciples de Saint-Simon, permettent, grâce à la bonne distribution des richesses dans le corps social, l'avènement d'une société industrielle et égalitaire. Travaillée par les métaphores du tissage et de la circulation, la symbolique du réseau en fait un moyen d'organisation du social. Conséquence de la comparaison du réseau aux nerfs et aux veines, celui-ci est envisagé en terme de capacité, de contenant et de connexions. C'est la circulation qui entretient les phénomènes de vie dans l'organisme. Dès lors, le développement des réseaux, et tout particulièrement des réseaux de communication, doit assurer, dans l'utopie technologique, la circulation des richesses et des informations, garantie d'une révolution politique.

Si le mot réseau n'est pas prononcé dans les textes de Proust, l'auteur n'en utilise pas moins deux de ses premières métaphores. Aux images de la circulation des flux et du tissage, qui déterminent bénéfiquement le réseau de communication dans la physiologie sociale de Saint-Simon et de ses disciples, Proust oppose l'eau sans cesse écoulée du tonneau des Danaïdes : la coupure arbitraire et l'indiscrétion des demoiselles, soit le bavardage, la panne et la surveillance. L'utilisation de figures féminines mythologiques pour évoquer les métaphores traditionnelles du réseau sexualisent le réseau téléphonique tout entier et non plus le seul objet "téléphone". Le temps "originel" du téléphone est marqué par l'imaginaire d'un réseau féminin, naturel et presque monstrueux.

Le téléphone, comme toute technique porteuse de promesses, est déceptif. Le lien fragile qu'il tisse semble toujours menacé par la panne, la surveillance et l'anarchie.

L'homme tend vers l'harmonie de la vie cosmique, c'est pourquoi la rupture est assimilée à une question de vie ou de mort. En tant que technique potentiellement performante, le téléphone est merveilleux, c'est-à-dire non humain. L'humain et la non réalisation de l'idéal resurgissent avec la panne et le silence de la machine de communication. Si "l'émerveillement et l'étonnement [ne] sont [que] les formes sublimées de l'effrot ${ }^{25 "}$ ", tout désenchantement fait renaître l'inquiétude. La définition du réseau qui le donne comme "ensemble de fils entrelacés, lignes et nouds ${ }^{200}$, renvoie explicitement à la technique du tissage, symbole de continuité qui s'oppose à la déchirure. Équivoque, la symbolique du tissage convoquée par Proust ne fait pas abstraction de la coupure et des ciseaux. En faisant référence aux Moires, l'auteur nous évoque les contingences du destin. "Les Filles de la Nuit t", les Moires qui fixent le destin, nous rappellent que nous restons soumis à la mort qui adviendra inexorablement et inscrivent au monde le primat féminin, le temps premier de la révélation et de la fertilité. Elles jouent également les intermédiaires entre les humains et le royaume des ombres, comme le suggère l'allusion de Proust au mythe 
d'Orphée.

Autre valeur du réseau inversée par Marcel Proust, la libre circulation des informations sur le réseau téléphonique apparaît difficilement observable avant 1934. La commutation manuelle réduit l'efficacité de l'exclusion du tiers. Ce désir d'échanger des informations en dehors de tout contrôle étatique est manifesté dans les débats qui précèdent la nationalisation du téléphone. Les partisans d'une libre exploitation formulaient clairement leurs craintes de voir l'État s'immiscer dans les conversations privées. Marcel Proust oppose donc l'indiscrétion des demoiselles à la promesse d'un réseau véhiculant librement des informations. Il les compare aux "ironiques Furies qui, au moment que nous murmurions une confidence à une amie, avec l'espoir que personne ne nous entendait, nous crient cruellement 'J'écoute "28'. Le réseau téléphonique de l'époque ne peut assurer 1" "immédiation" nécessaire à l'avènement de la société égalitaire, et il échoue à signifier "l'association universelle $e^{29 "}$. La réduction de la distance qui sépare les deux interlocuteurs mis en présence par le réseau téléphonique ne réduit nullement les distances sociales ainsi que l'espérait Michel Chevalier $^{30}$. Les trois Furies représentent la perpétuelle surveillance, mais personnifient également la culpabilité. Le téléphone pouvant être un vecteur de séduction, l'intervention des demoiselles dans les confidences échangées par les interlocuteurs évoque le possible surgissement d'un sentiment de culpabilité.

Le bon fonctionnement du réseau implique que la décision n'émane ni d'un centre despotique, ni anarchiquement en ses extrémités. Or, l'auteur reproche au réseau téléphonique de mal organiser les flux de paroles véhiculés par les câbles du téléphone. La figure mythologique du tonneau des Danaïdes ${ }^{31}$ évoque à juste titre cet écoulement incontrôlé et désordonné des sons dans les appareils téléphoniques. Pour avoir égorgé leurs époux durant leur nuit de noce, les Danaïdes furent condamnées par les juges des morts à demeurer aux Enfers où elles rempliront à jamais un tonneau criblé de trous. En refusant d'accomplir un acte naturel dans des conditions légitimes, l'homme (ou la femme) se condamne à une absurdité sans fin (répéter continuellement les mêmes actes stériles). L'écoulement ininterrompu des sons et le contenu des paroles échangées témoignent de la vacuité des préoccupations féminines. L'usage féminin du téléphone est expressif, les confidences échangées sont nécessairement futiles. Il s'oppose à l'usage masculin, instrumental, qui vise l'échange d'informations ou le commandement. En invoquant ce mythe, Proust semble condamner le travail féminin tout comme l'oisiveté de la femme des classes élevées. Plutôt que de se soumettre à la "loi naturelle" qui les destine au mariage et à la reproduction, les employées du téléphone consacrent leur jeunesse à une activité stérile : transmettre des conversations futiles, voire condamnables. Plutôt que de trouver à s'occuper dans leurs foyers, les riches oisives se livrent au "téléphonage" et incarnent le parasitisme dont souffrent les classes laborieuses.

Les demoiselles du téléphone évoquent à l'auteur les prophétesses de l'Antiquité. Comme elles, les téléphonistes prêtent leur corps afin que s'établisse une communication verticale entre 
dieux et hommes. La forme creuse de la femme en fait un canal privilégié. Des profondeurs de la terre ${ }^{32}$, le souffle divin traverse son corps: "le support corporel du lien oraculaire est fantasmé : il ne donne aucune prise à la vue mais seulement à l'oreille ${ }^{33}$ ". De même, le téléphone transmet la voix en l'absence du regard. Les interlocuteurs, presque aveuglés, "tâtonnent dans [des] ténèbres vertigineuses ${ }^{34}$." Soumis aux caprices de sombres divinités, le téléphone ne peut répondre aux promesses d'une technique porteuse d'égalité. Les "messagères de la parole $e^{35}$ " évoquent probablement Iris, l'arc en ciel reliant le ciel et la terre, divinité mineure du Panthéon grec et messagère des dieux qui s'efface progressivement au profit d'Hermès. Si les deux divinités partagent de nombreuses caractéristiques telles que l'intelligence et la rapidité, Hermès parcourt la terre, pratique des échanges et ne se limite pas à la transmission des messages de Zeus. D'Isis à Hermès, la communication évolue. Madeleine Campana convoque elle aussi la figure de l'oracle, comparant les demoiselles du téléphone à des "prêtresses [...] émettant dans leurs cornets d'interminables confessions" dans "une salle aussi grande que la nef d'une cathédrale ${ }^{360}$.

Selon Catherine Bertho, les notables de la troisième République, responsables du téléphone, veillent à ce qu'il n'y ait aucune dépense inutile. La parole étant un luxe, elle n'est accessible qu'à quelques privilégiés. À défaut d'une technique performante, financée par l'État ct accessiblc à tous (comme apparaît le télégraphe à l'époque), le téléphone nécessite un investissement financier personnel. Au sein même des abonnés, s'instaure une hiérarchie : les numéros prioritaires sont représentés par des lampes de couleur rouge.

Le constat d'une référence répétée de l'auteur à des divinités féminines pour évoquer les demoiselles du téléphone nous a suggéré plusieurs questionnements. Si le téléphone fonctionne à l'électricité, il se charge des promesses de celle-ci. Pourtant, le téléphone des années 1880 à 1914 est déceptif. À ce stade primitif et féminin, oral, dirait Pierre Musso ${ }^{37}$, doit succéder un stade technique, masculin qui ordonnera le réseau et limitera les défaillances du système.

Toutes les mythologies commencent par la défaite des mères ${ }^{38}$ ou du matriarcat ${ }^{39}$. À l'origine, il y a deux mères dans la cosmogonie grecque : Terre et Nuit, issues de l'Abîme primordial. La seconde engendre sa descendance par scissiparité, loin du principe masculin, tandis que Terre engendre Rhéa, mère des Olympiens. Une dissymétrie relève de ces deux modes de procréation. La procréation par scissiparité date des premiers commencements et évoque l'idée menaçante d'une féminité close sur elle-même. Les figures mythologiques convoquées par Proust s'inscrivent toutes en opposition à la fonction "naturelle" de la femme, et évoquent des sociétés féminines refermées sur elles-mêmes. Les Moires sont issues de Nuit, les Furies naissent du sang d'Ouranos versé sur Terre par l'émasculation perpétrée par Chronos. Ces personnages mythologiques sont placés dans des contrées retirées, les Enfers, loin des hommes qui ignorent lcur aspect physique. Ceux-ci les imaginent hybrides, moitié femme moitié animal, autres sphinges symbolisant les puissances naturelles monstrueuses que la logique masculine d'Edipe ou la science prométhéenne terrassent. Oralité qui 
écarte la vue, verticalité du lien, métaphore des Enfers, le réseau téléphonique s'apparente plus aisément à la figure inquiétante de la pieuvre qu'à la représentation d'un réseau artificiel vecteur de lumières et de fraternité. La figure de la femme hybridée à l'animal témoigne de la perversion de la rencontre femme/machine. $\mathrm{Si}$ la pieuvre symbolise généralement des réseaux financiers et industriels occultes ${ }^{40}$, le réseau téléphonique semble permettre une conspiration des femmes entre elles, une séduction généralisée, s'insinuant jusqu'au cœur du foyer et susceptible d'ébranler l'autorité de l'époux et du père.

Proust dessine, en définitive, un imaginaire équivoque du téléphone des origines. En convoquant à la fois les images bénéfiques de l'électricité, la symbolique ambivalente du tissu et des symboles terrifiants de la puissance maléfique de la nature féminine, il s'éloigne de l'utopie technologique et de la dénonciation formelle du potentiel immoral de l'objet technique. L'usage expressif du téléphone l'inspire, plus encore que le "paradigme de l'échange égalitaire ${ }^{4 l "}$.
N

1. J. Noiray.- Le romancier et la machine. L'image de la machine dans le roman français 1850-1900. Tome I : Émile Zola.- Librairie José Corti, Paris 1982 , p. 421.

2. M. Campana.- Op. Cit.- p. 248.

3. Ibid.- p.33.

4. Article publié dans Le Journal des téléphones du 25 janvier 1924 et reproduit dans J. Bouvier.- Histoire des dames employées dans les PTT de 1714 à 1929.- Paris, PUF, 1930, p. 184-185.

5. M. Campana.- Op. Cit.- p. 34.

6. J. W. Scott.- Op. Cit.- p. 481.

7. M. Perrot.- "De la nourrice à l'employée. Travaux de femmes dans la France du XIX" siècle".- Le Mouvement social, $\mathrm{n}^{\circ}$ 105, Éditions ouvrières, 1978.

8. La scène du téléphonage est reprise dans l'article "Journées de lecture", publié le 20 mars 1907 dans Le Figaro, et que nous pouvons lire au chapitre "communication et littérature" dans le Dictionnaire critique de la communication (L. Sfez.- Dictionnaire critique de la communication.- PUF, 1993, Tome I, p. 68).

9. "Les fileuses" dans P. Brunel (dir.).- Dictionnaire des mythes littéraires.- Éd. du Rocher, 1988.

10. Affiche Singer reproduite dans G. Duby et $M$. Perrot (dir).- Op. Cit.- p. 366.

11. J. Noiray.- Op. Cit. Tome I.- p. 406.

12. M. Campana.- Op. Cit.- p. 203.

13. M. Proust.- Ibid.- p. 212.

14. Association des abonnés au téléphone.- Une honte nationale, l'anarchie téléphonique.- La Rochelle, Texier, 1909.

15. L. Sfez. (dir.).- Dictionnaire critique de la communication.- Tome I, p. 68.

16. J. Bouvier.- Op. Cit.-p. 180. 
17. M. Campana.- Op. Cit.- p. 134.

18. C. Bertho.- Op. Cit.- p. 239.

19. Voir à ce propos M. Foucault.- Histoire de la sexualité. Tome I : la volonté de savoir.- Paris, Gallimard, coll. "Tel", 1976.

20. C. Bertho.- Op. Cit.- p. 224, pp. 236-237.

21. M. Proust.- Op. Cit. Tome I.- p. 209.

22. "Journées de lecture" publié dans Le Figaro du 20 mars 1907.

23. Op. Cit.- p. 355.

24. Voir à ce propos L. Sfez.- Technique et idéologie.Éd. du Seuil, coll. "La couleur des idées", 2002, p. 143. 25. P. Diel.- Op. Cit.- p. 92.

26. P. Musso "La symbolique du réseau" dans revue Quaderni n ${ }^{\circ}$ 38, CREDAP, Université Paris I Sorbonne, Printemps 1999, p. 72.

27. M. Proust.- Op. Cit. Tome I.- p. 212.

28. Ibid, p. 209.

29. "Le système de la Méditerranée", article de Michel Chevalier publié dans Le Globe du 12 février 1832.

30. Cité dans "La symbolique du réseau".- Op. Cit.p. 82.

31. M. Proust.- Op. Cit.- p. 209.

32. "Il m'a semblé que cette voix clamait des profondeurs d'où l'on ne remonte pas" M. Proust.Op. Cit.- p. 209.

33. "Antiquité" dans L. Sfez (dir.).- Dictionnaire critique de la communication.- PUF, 1993, p. 1553.

34. M. Proust.- Op. Cit.- p. 209.

35. Ibid.- p. 212.

36. M. Campana.- Op. Cit.- p. 62.

37. P. Musso.- Télécommunication et Philosophie des réseaux. La postérité paradoxale de Saint Simon.- PUF, coll. La politique éclatée, Paris, 1997, p. 231.

38. Voir la lecture que C. Ramnoux fait de la Théogonie d'Hésiode dans Mythologie ou la famille olympienne.Brionne, Gérard Monfort, édition de 1982.

39. Pour Engels, on peut supposer qu'il existait dans un temps primitif un véritable règne des femmes. Le passage du matriarcat au patriarcat lui apparaît comme "la grande défaite historique du sexe féminin" cité dans S. de Beauvoir, Le deuxième sexe. Tome II.- Paris, Gallimard, coll. "Folio Essais", édition de 1976, p. 121. 40. P. Musso.- Critique des réseaux.- Paris, PUF, coll. La politique éclatée, avril 2003, p. 260.

41. P. Musso.- Télécommunication et Philosophie des réseaux. La postérité paradoxale de Saint-Simon.- PUF, coll. “La politique éclatée”, Paris, 1997, p. 231. 